\title{
ANÁLISIS DE LOS ESTABLECIMIENTOS COMERCIALES EN LA CIUDAD DE VILLAVICENCIO FRENTE A LOS IMPACTOS NEGATIVOS DE TIPO SOCIOECONÓMICO
}

\author{
ANALYSIS OF COMMERCIAL ESTABLISHMENTS IN THE CITY OF \\ VILLAVICENCIO AGAINST NEGATIVE SOCIO-ECONOMIC IMPACTS
}

\section{Castro Garzón, Hernando'}

Universida de los Llanos, Villavicencio,

Colombia

\section{Hernández Criado, Juan Carlos²}

UFPSO,Universidad Francisco de Paula

\section{Santander Ocaña}

\section{Sánchez Céspedes, Juan Manuel ${ }^{3}$}

Universidad Distrital Francisco José de Caldas, Bogotá, Colombia

\section{RESUMEN}

Los establecimientos comerciales son los entes en los que se soporta la economía de Villavicencio, el Centro histórico de la ciudad se caracteriza por agrupar comerciantes dedicados a distintas actividades, a su vez

$1 \quad$ Profesor, Escuela de Administración y Negocios. Facultad de Ciencias Económicas, Universidad de los Llanos, Villavicencio, Colombia. Correo electrónico: hcastro@ unillanos.edu.co. ORCID: https://orcid.org/0000-0003-12990489

2 Docente Investigador. Universidad Francisco de Paula Santander Ocaña, Colombia. Email: jchernandezc@ ufpso.edu.co. ORCID: https://orcid.org/0000-0002-45914047

3 Profesor Titular. Facultad de Ingeniería. Universidad Distrital Francisco José de Caldas. Bogotá, Colombia. Correo electrónico: imsanchezc@udistrital.edu.co ORCID: https://orcid.org/0000-0001-9101-2936

funciona como acopio de mercancías para la venta al detal como al por mayor, convirtiéndose en despensa regional. Por eso, se consideró necesario realizar una caracterización de los establecimientos para medir el impacto de los factores socioeconómicos que los afectan para que así se puedan formular estrategias para mitigar los efectos que estos producen.

Se aplicó el método cuantitativo, con un diseño tipo descriptivo- no experimental, se consideró una muestra significativa para su abordaje, acometiendo a los individuos de interés a través de una encuesta con preguntas de única respuesta y de escala numérica que se aplicó a los propietarios o administradores de los 
establecimientos considerados, posteriormente se tabuló a través de la generación de una base de datos a través de Excel para facilitar el análisis estadístico.

En las conclusiones se resaltó que los comerciantes percibían el factor económico el de mayor impacto negativo a causa de la crisis por la pandemia; entre los conceptos sociales, se determinó que la inseguridad a pesar de ser un factor de vieja data los sigue afectando, también propusieron una mayor participación por parte del gobierno seccional, estrategias publicitarias para el centro, programas de rehabilitación para los habitantes de calle y reubicación de los vendedores ambulantes.

\section{ABSTRACT}

The commercial establishments are the entities in which the economy of Villavicencio is supported, the historic center of the city is characterized by grouping merchants dedicated to different activities, in turn it works as a collection of merchandise for retail sale and wholesale, becoming a regional pantry. For this reason, it was considered necessary to carry out a characterization of the establishments to measure the impact of the socioeconomic factors that affect them so that strategies can be formulated to mitigate the effects they produce.

The quantitative method was applied, with a descriptive-not experimental type design, a significant sample was considered for its approach, engaging the individuals of interest through a survey with single-answer questions and a numerical scale that was applied to the owners or administrators of the establishments considered, later it was tabulated through the generation of a database through Excel to facilitate statistical analysis.

The conclusions highlighted that merchants perceived the economic factor as the one with the greatest negative impact due to the crisis caused by the pandemic; Among the social concepts, it was determined that insecurity despite being a factor of old date continues to affect them, they also proposed greater participation by the sectional government, advertising strategies for the center, rehabilitation programs for street dwellers and relocation of street vendors.

\section{PALABRAS CLAVE}

comercio, pandemia, sostenibilidad.

\section{KEYWORDS}

trade, pandemic, sustainability.

\section{INTRODUCCIÓN}

El sector de comercio al por mayor y por menor, se caracteriza por tener mayor participación en toda la jurisdicción de la Cámara de Comercio de Villavicencio. En el 2019 el departamento del Meta en el sector del comercio de manera general aportó al PIB nacional $11.3 \%$, de acuerdo con cifras del (DANE, 2019), Villavicencio se ha caracterizado por ser un punto de referencia comercial para los demás municipios de la región Orinoquia, porque es donde se concentra la mayoría de grandes superficies mayoristas, como las minoristas, ubicadas principalmente en el centro de la ciudad; el sector del comercio también tiene una alta participación en el PIB, más exactamente en la rama de hoteles y restaurantes, donde aporta el $43 \%$ de empleos a la ciudad según la (Cámara de Comercio de Villavicencio, 2017), al ser la capital del departamento del Meta y la entrada principal hacia el departamento, se convierte en fuente de acopio para la región.

En todos los sectores económicos existen factores que afectan negativa o positivamente la funcionalidad de los negocios o empresas, para el caso de Villavicencio haciendo referencia al barrio centro y al sector del comercio, algunos de estos factores que afrontan según la (Cámara de Comercio de Villavicencio, 2017) pueden ser 
la informalidad, inseguridad, pico y placa, valor de arrendamientos, habitantes de calle, reforma tributaria entre otros. Actualmente, no solo la ciudad sino el país enfrenta la pandemia que ha ocasionado un constreñimiento en la economía, a nivel nacional el sector de comercio al por mayor y al por menor presentó un crecimiento para mayo del presente año de $-15,2 \%$ (DANE, 2021) con una variación del $-0,1 \%$ frente a marzo del mismo año, este resultado ocasionado por la crisis de la pandemia.

Otro rasgo es que la informalidad, se puede apreciar desde dos perspectivas, la primera, que los vendedores informales que deambulan por las calles del centro de la ciudad o que en ocasiones se instalan de forma permanente en un lugar estratégico, que para (Orduz \& Dimas, 2019) estos trabajadores en su mayoría encuentran mejores condiciones laborales en las calles que en un empleo formal, en su investigación afirmaron que pueden generar mayores ingresos y no cumplir horario; la otra, en que las propias empresas contraten de manera informal o realicen sus actividades sin previa formalización ante las entidades correspondientes.

Port otro lado, en cuanto a la inseguridad en los establecimientos de comercio, en el año 2019 se presentaron 790 casos de hurto a comercio en Villavicencio, pero en los últimos años se evidencia una disminución de este factor desde 2017 donde presentó una tasa de 190.7 y en el 2019 en 149.7 (Departamento Nacional de planeación, 2020), en 2 años la tasa de hurto disminuyó 41 puntos.

Mas aun, en lo expuesto como la medida de pico y placa en la ciudad de Villavicencio, dado el creciente flujo vehicular especialmente de motocicletas que llegó a contar con el $79 \%$ de los matriculados en la ciudad, ocasionando que la congestión llegara al 90\% (Suárez, 2018). Al inicio de la medida se estableció un polígono que afectaba directamente el centro de la ciudad impactando el comercio allí asentado; actualmente la medida está rigiendo en toda la ciudad.

Un efecto macroeconómico, pero de incidencia considerable son los cambios tributarios, el aumento del IVA del $16 \%$ al 19 en la anterior reforma y actualmente con la nueva propuesta de aumento de IVA; según (Pintor \& Guevara, 2019) hubo alza de varios productos, incluso de la canasta familiar, pero que de acuerdo a las condiciones del mercado presentaron alza en su valor.

Por otra parte, la presencia de las medidas de contención para hacer frente a la pandemia ha ocasionado efectos negativos para la economía, las medidas que estableció la Alcaldía de Villavicencio para evitar el aumento en la ocupación de los centros hospitalarios como los toques de queda, confinamientos obligatorios en fines de semana, pico y cédula para ingresar a bancos y establecimientos de comercio entre otras medidas de carácter plenamente sanitario pero que ocasionaron efectos para los comerciantes de Villavicencio (Instituto Nacional de Salud, 2020).

Por lo anteriormente mencionado, se realizó un estudio donde se pudo apreciar los factores que afectan las dinámicas socio económicas de un sector clave para la región sus condicionamientos, así como las posibles estrategias que coadyuven a dinamizar una zona de alto impacto para la ciudad.

\section{METODOLOGÍA}

Para la investigación se realizó bajo el tipo de investigación descriptivo- no experimental, ya que se enfocó en mostrar los resultados que permitieron la identificación de los factores económicos y sociales que más afectan al comercio; a su vez proporcionó información de las características de los establecimientos comerciales ubicados en 
la zona de estudio; también se definió un tratamiento cuantitativo de la información.

La población que se determinó para el estudio se estimó en 39.300 establecimientos de comercio (Camára de Comercio de Villavicencio, 2017), en cuanto al centro de Villavicencio se estima que puede existir alrededor de 1500 locales productivos. La muestra que se tomó como referencia fue la calle de los cielos abiertos por la variedad de negocios agrupados allí, se tomó como muestra un total de 105 establecimientos comerciales, en los que se encontraron 13 de maquillaje y accesorios, 8 restaurantes, 5 pañaleras o jugueterías, 8 servicios médicos, 41 de ropa y calzado, 10 de tecnología, 1 agencia de viajes y 19 cacharrerías o papelerías.

El instrumento que se utilizó fue un cuestionario quesedividió entrespartes, la primerainformación general que ayudó a la caracterización de los establecimientos; la segunda que tiene que ver con los factores económicos y sociales que podían llegar a afectar a los comerciantes; y la tercera las posibles estrategias para reducir los efectos negativos de los factores.

\section{ANÁLISIS DE RESULTADOS}

Se presentan acordes a tres elementos de análisis descritos a continuación, caracterización, factores de afectación y por último estrategias de mitigación.

\section{Caracterización de los establecimientos de comercio del Centro de Villavicencio}

$\begin{array}{llll}\text { Antigüedad del } & \text { El 52\% tiene entre } 3 \text { y } 4 & \text { El 23\% tiene menos de } & \text { El 25\% tiene más } \\ \text { establecimiento } & \text { años } & 2 \text { años } & \text { de } 5 \text { años }\end{array}$

$\begin{array}{llll}\text { Tipo de local } & \text { El 95\% es arrendatario } & \text { El 5\% posee local propio } & \\ \text { Cantidad de empleados } & \begin{array}{l}\text { El 78\% tiene de 1 a } 3 \\ \text { empleados }\end{array} & \begin{array}{l}\text { El 17\% de 4 a } 5 \\ \text { empleados }\end{array} & \begin{array}{l}\text { El } 5 \% \text { de } 6 \text { a } 10 \\ \text { empleados }\end{array} \\ \text { Tipo de cliente } & \begin{array}{l}\text { El 48\% atiende cliente } \\ \text { minorista }\end{array} & \begin{array}{l}\text { El 52\% atiende a } \\ \text { minoristas y mayoristas }\end{array} \\ \text { Puntos de venta } & \text { El 14\% tiene más puntos } & \text { El 86\% no tiene más } \\ \text { de venta } & \text { puntos de venta } & \end{array}$

Tabla 1 Principales características de los establecimientos de comercio del barrio Centro de Villavicencio. Los autores (2021)

\section{ANTIGÜEDAD DEL ESTABLECIMIENTO.}

Un poco más de la mitad $52 \%$ de los negocios "de los cielos abiertos" se encuentra entre 3 y 4 años de antigüedad, apenas un $25 \%$ tiene más de 5 años de trayectoria en el mercado, lo cual es positivo porque la mayoría superan los 3 años de vida, demostrando su pervivencia en el mismo.

\section{TIPO DE LOCAL.}

El $95 \%$ es decir la mayoría de los comerciantes del sector, desarrolla sus actividades comerciales en locales que no son de su propiedad, tan solo el $5 \%$ posee uno propio que probablemente le permita tener un poco más de solvencia económica frente a los establecimientos que pagan arriendo, cabe resaltar que el valor del 
arriendo depende de la ubicación, la planta y el tamaño del local.

\section{CANTIDAD DE EMPLEADOS}

De acuerdo al número de empleados con el que disponen los establecimientos, el $78 \%$ cuenta con entre 1 y 3 empleados, en algunos casos se observó durante el trabajo de campo que el dueño era el único presente, en locales de venta de ropa o cacharrerías de superficies mayores se encontraban con un número superior, se estableció que no se encontraron más de 10 empleados por almacén.

\section{TIPOS DE CLIENTE.}

Se determinó que la variación en cuanto al tipo de clientes, al por menor o al mayoreo fue del 4 $\%$, estableciendo que la zona de estudio es de acopio para comercio al detal.

\section{PUNTOS DE VENTA.}

Para una empresa es necesario expandirse en el transcurrir del tiempo, ya sea por demanda del cliente, ampliación de inventario o estrategia comercial; en el barrio Centro se evidenció que el $14 \%$ de los comerciantes tienen otros puntos de venta mientras que el $86 \%$ solo cuenta con el punto dentro de la calle de los cielos abiertos.

\section{Factores económicos que afectan a los establecimientos de comercio del centro de Villavicencio}

\section{Impacto negativo de factores económicos}

\section{$70 \%$}

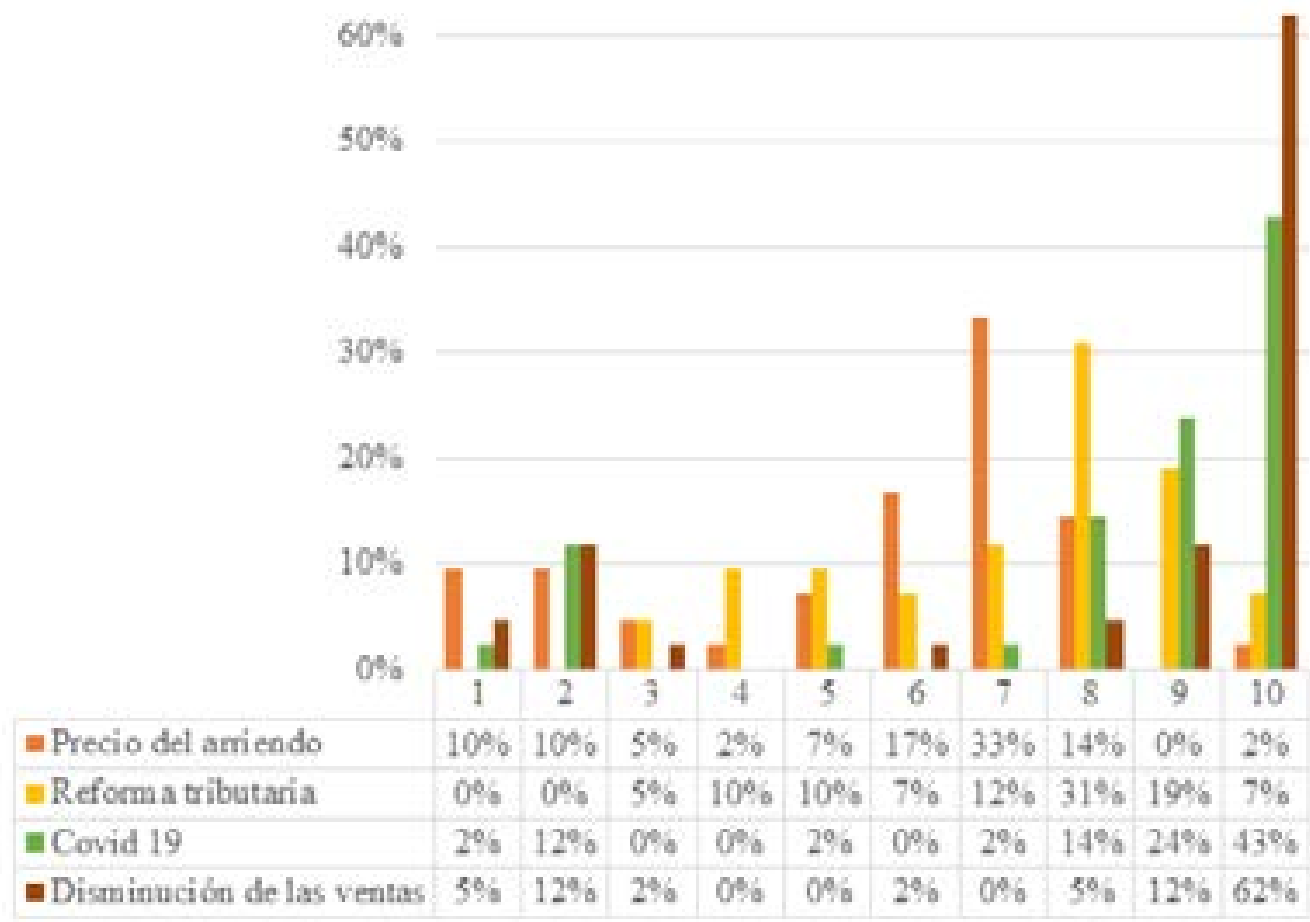

Figura 2 Factores económicos que afectan el Centro de la ciudad de Villavicencio.

Los autores (2021) 
Para este análisis se tuvo en cuenta una escala de medición de 1 al 10, donde el menor impacto era el $1 \mathrm{y}$ el mayor el 10.

El primer factor es el precio del arriendo donde el $33 \%$ de los establecimientos calificaron el factor en 7 , siendo este el nivel de impacto con mayor número de puntos; para el segundo factor de la reforma tributaria el nivel de la escala con mayor puntuación fue el 8 , con $31 \%$ de encuestados; el factor del COVID 19 obtuvo un nivel de impacto de 10 con el $43 \%$ de los establecimientos; y el último factor establecido es la disminución de las ventas, con un mayor impacto percibido, se posicionó en el nivel 10 con una incidencia del $62 \%$; siendo el principal factor económico que los afecta es la disminución de ventas, seguido del COVID 19, la reforma tributaria y el precio del arriendo.

\section{PRECIO DEL ARRIENDO}

Los comerciantes afirmaron que los precios del arriendo aumentan cada año pero que estos aumentos han sido desmesurados, es decir, en la mayoría de los locales no se aplica el (artículo 20 de la Ley 820 de 2003) en el se establece los porcentajes anuales de los cánones del arriendo debe aumentar de acuerdo a la inflación, es decir que para 2021 debería ser de 1,6\%. En la gráfica se pudo determinar que los locales que se han visto perjudicados por el precio del arriendo son un $64 \%$ por los altos precios y un $7 \%$ por la escasez de dinero a causa de la pandemia.

\section{REFORMA TRIBUTARIA}

Los comerciantes de Centro de Villavicencio están inconformes con la última reforma tributaria, el $55 \%$ afirmó que es un abuso del gobierno, el $17 \%$ que los ingresos que perciben no son suficientes para pagar impuestos y el $2 \%$ que se le dificulta contratar un contador, el $26 \%$ no se ha visto perjudicado por la reforma tributaria. Al cruzar esta pregunta con el nivel de ingresos percibidos se evidenció que la mayoría de establecimientos que no se ve perjudicado por este efecto son los locales pequeños mientras que los grandes afirmaron en su mayoría que era un abuso del gobierno; estableciendo en (Barría, 2021) "las distintas visiones sobre quiénes deben pagar la cuenta fiscal que ha dejado la pandemia hacen que el camino de Colombia sea difícil", ya que los empresarios no quieren asumir esta responsabilidad luego de la crisis vivida durante el 2020.

\section{COVID 19.}

La medidas para el control de la pandemia impacto a todos los empresarios y comerciantes; el bajón económico a causa del desempleo o inactividad de muchos colombianos, según (DANE, 2021) "Si se comparan los resultados de mayo de 2021 con los de mayo de 2019 se evidencia una disminución de $10,2 \%$ en las ventas del comercio minorista y una caída en el personal de 4,8\%"; el resultado del DANE cruzado con el estudio realizado, evidencia que el COVID 19 si ha generado efectos negativos, ya que el $43 \%$ afirmó disminución de ventas y el $31 \%$ presentó pérdidas para su negocio.

\section{DISMINUCIÓN EN LAS VENTAS}

El COVID 19 es el principal factor que afecta las ventas, ya que el $74 \%$ lo afirmó, este factor esta correlacionado directamente, según él (Banco Mundial, 2021) "en una cuarta parte de las empresas las ventas disminuyeron un $50 \%$, en promedio disminuyeron el $27 \%$ ", presentando una radiografía de la situación mundial. 


\section{Factores sociales que afectan a los establecimientos de comercio del Centro de Villavicencio}

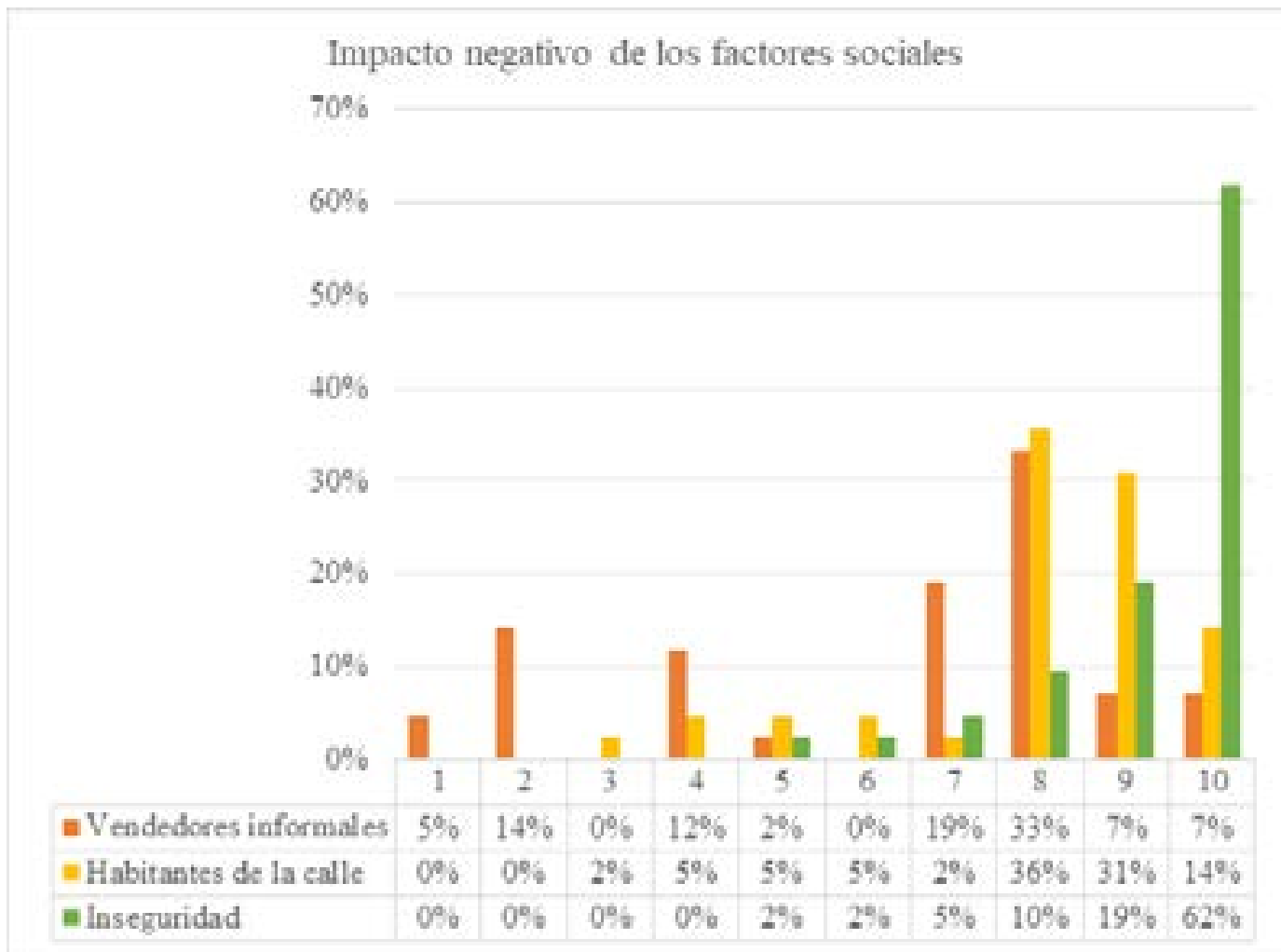

Figura 3 Factores sociales que afectan el Centro. Los autores (2021)

Para este análisis se tuvo en cuenta una escala de medición de 1 al 10, donde el menor impacto era el 1 y el mayor el 10.

Los vendedores ambulantes presentaron una calificación de nivel 8 en la escala de medición con un $33 \%$ de los establecimientos comerciales; el factor negativo de los habitantes de la calle obtuvo en la escala un nivel de calificación 8 con $36 \%$ siendo este el más alto, seguido del 9 con $31 \%$ de los comerciantes; en cuanto a la inseguridad esta obtuvo la calificación más alta de los tres factores medidos, ya que en una escala del 1 al 10 , obtuvo 10 con el $62 \%$ de los establecimientos comerciales.

\section{VENDEDORES INFORMALES}

Para el sector estudiado, se encontró que la responsabilidad en que sus ventas disminuyan en un $31 \%$, seguido de que no pagan impuestos, generando competencia ya que pueden bajar precios con $19 \%$ y el $7 \%$ que afirma que ocupan el frente y restringe el acceso a sus clientes, pero el $43 \%$ afirmó no sentirse perjudicado por los vendedores informales. El comercio informal produce problemáticas con la ocupación del espacio público y hasta competencia desleal, según (Caícedo, Calvache, \& Jiménez, 2020) "el trabajo informal es una parte fundamental en la economía de muchos hogares colombianos, aporta a la reducción de la tasa de desempleo" (p.75).

\section{HABITANTES DE CALLE}

Para los comerciantes analizados, los habitantes de calle los han perjudicado de distintas maneras, el $62 \%$ afirmó que la mendicidad incomoda a sus clientes, al $26 \%$ los afecta el robo de mercancía y a los clientes, el $12 \%$ afirmó que los habitantes de la calle causan mala presentación para su negocio. Para "la Reducción de fenómeno de habitabilidad de calle requiere de la participación 
activa y comprometida de ... las organizaciones gubernamentales como la comunidad, el sector empresarial y los gerentes sociales manteniendo un compromiso moral con la transformación" (Rossiasco \& Franco, 2018, P.60).

\section{INSEGURIDAD}

Los propietarios de los comercios intervenidos manifestaron que se han visto perjudicados por la inseguridad, el $90 \%$ afirmó ser afectados por el fenómeno, la inseguridad del centro es propiciada por que alrededor de este se encuentran barrios peligrosos "ollas" las cuáles hacen que la inseguridad aumente (Franco, 2014), algunas de estas "ollas" son los barrios San José, Villa Julia, Industrial entre otros.

\section{Estrategias propuestas por los comerciantes del centro deVillavicencio para la mitigación del impacto negativo de los factores económicos que los afectan}

\section{Proponga una estrategia para el mejoramiento de los factores económicos que afectan a los comerciantes}

Estrategia de mitigacion de efectos económicos por el Covid

Más publicidad para el comercio local

Más seguridad y cuidados frente al COVID

Más apoyo de la Alcaldia y gobiemo en general

No sabe no responde

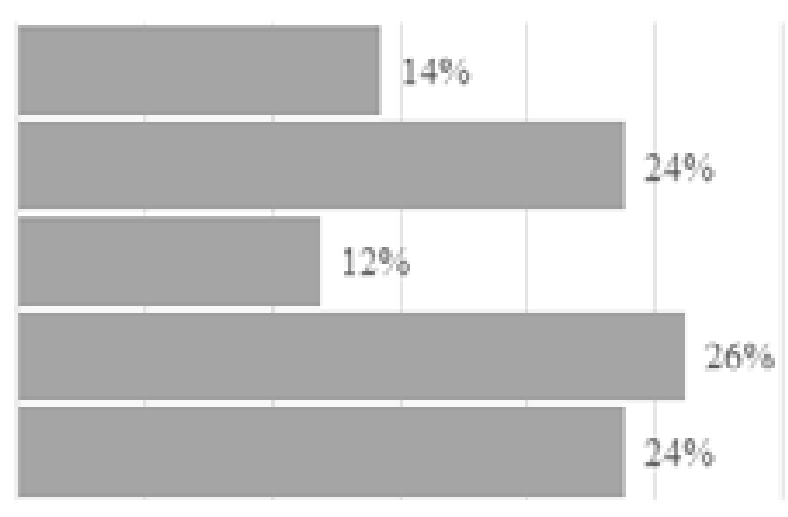

Figura 4 Estrategias para mitigar los factores económicos negativos. Los autores (2021)

Los dueños de los establecimientos de comercio al por mayor y detal propusieron estrategias para mejorar los factores económicos que se midieron con anticipación en el estudio, donde el $24 \%$ se mostró indiferente en proponer una estrategia, algunos manifestaron que no se tomaban en cuenta, el $26 \%$ dijo que se necesitaba más apoyo de la Alcaldía y gobierno en general, el otro $24 \%$ manifestó que se necesitaba aumentar la publicidad del comercio local para atraer clientes, el $14 \%$ que era necesario mitigar los efectos económicos de la pandemia y el $12 \%$ que se necesitaba más seguridad y cuidados frente al COVID 19. 


\section{Estrategias propuestas por los comerciantes del centro de Villavicencio para la mitigación del impacto negativo de los factores económicos que los afectan}

\section{Proponga una estrategia para el mejoramiento de los factores sociales que afectan a los comerciantes}

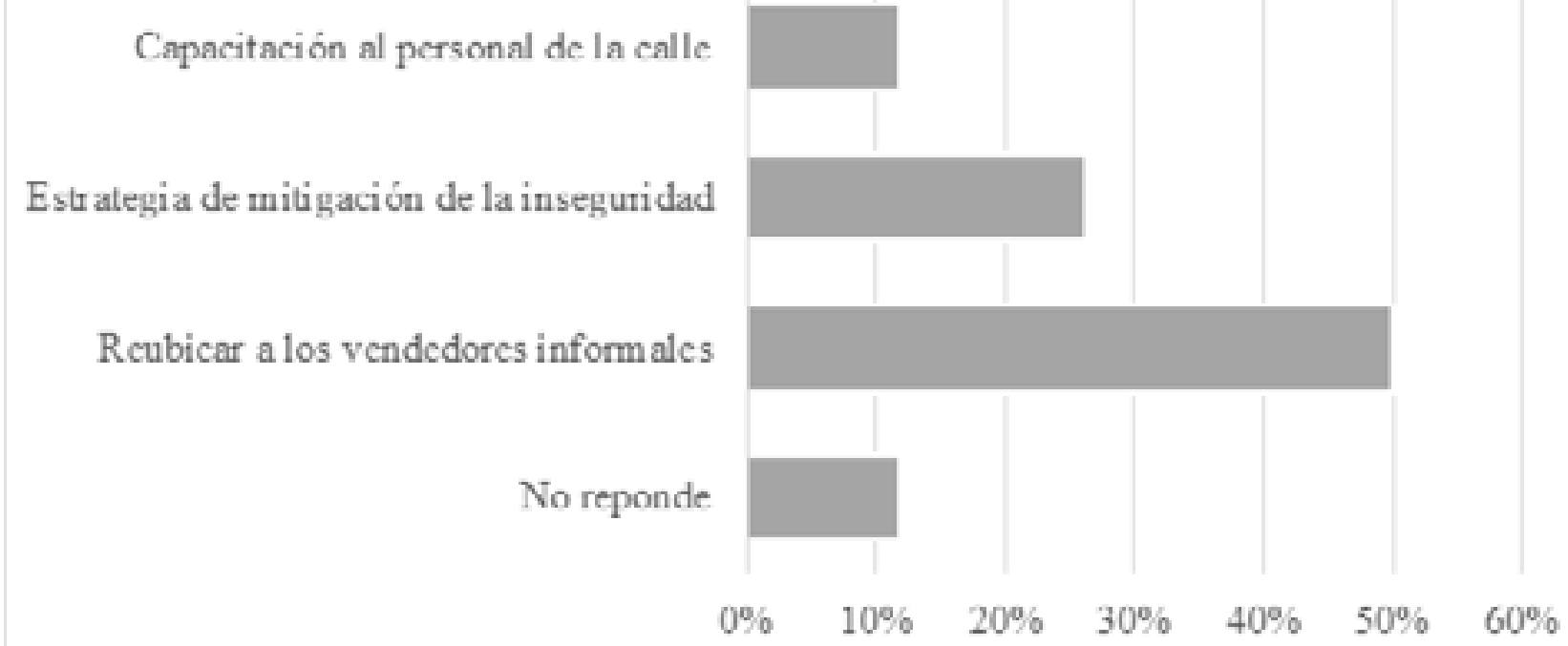

Figura 5 Estrategias para mitigar los factores sociales negativos. (Los autores 2021)

La respuesta establecida para disminuir el impacto de los efectos de tipo social fue propuesta por la mitad de los comerciantes encuestados, de reubicar a los vendedores informales, seguido del $26 \%$ que propuso mitigar la inseguridad, el $12 \%$ propuso capacitar el personal de la calle tanto vendedores ambulantes como habitantes de calle, y el $12 \%$ prefirió no responder.

\section{CONCLUSIONES}

El centro de Villavicencio es un lugar que reúne a establecimientos de todo tipo se ha convertido en un epicentro de llegada para compradores, el comercio de la zona vende sus productos a los distintos clientes que pueden ser mayoristas 0 detallistas, convirtiéndose así en un eje para la economía del municipio.

En cuanto a la caracterización de los establecimientos de comercio, se concluyó que: la antigüedad de los establecimientos proporciona una idea de la estabilidad que han logrado estos en el mercado, mostrando que aquellos establecimientos con más de 3 años tienen una estabilidad financiera dado que han logrado superar los efectos negativos de la pandemia.

La propiedad de los establecimientos es escaza, pues solo el $5 \%$ cuenta con local propio mientras que un $95 \%$ paga arriendo, el $64 \%$ de los comerciantes afirmó que el precio de los arriendos era elevado, que el valor de este depende del tamaño y ubicación del local, de acuerdo a la información proporcionada se estableció que el valor del arriendo en algunos casos no están acorde a la inflación como dice la ley, además que se dificulta la compra de propiedad por la alta valorización de la zona comercial. 
El número de empleados con el que cuentan los establecimientos de comercio ayudan a definir el tamaño de la empresa, el $78 \%$ tiene entre 1 y 3 empleados donde se encontró que en muchas ocasiones el mismo propietario es el único empleado, no se encontraron establecimientos con más de 10 empleados, lo cual llevó afirmar que los establecimientos de la cuadra de "los cielos abiertos" seleccionada como muestra son microempresas.

Los comerciantes establecieron que para mejorar los factores económicos que afectan el centro falta más apoyo de la Alcaldía municipal y del gobierno nacional para solventar los efectos negativos de la pandemia y que se necesita más publicidad para impulsar el comercio local; para los factores sociales se propuso reubicar a los vendedores, capacitar al personal de la calle y aumentar la seguridad en el sector.

\section{AGRADECIMIENTOS}

Por su incesante colaboración y participación en el estudio a Brigitte Alejandra Gutiérrez Cuadrado y Diego Alfonso Castro Perilla.

\section{REFERENCIAS BIBLIOGRÁFICAS}

Artículo 20 de la Ley 820 de 2003. (s.f.). Secretaria Senado. Obtenido de http:// www.secretariasenado.gov.co/senado/ basedoc/ley_0820_2003.html

Banco Mundial. (17 de 02 de 2021). Banco Mundial. Obtenido de https:// www.bancomundial.org/es/news/ infographic/2021/02/17/how-covid-19isaffecting-companies-around-the-world
Barría, C. (13 de 05 de 2021). Protestas en Colombia: por qué es tan importante una nueva reforma tributaria en el país. BBC News Mundo, págs. https://www. bbc.com/mundo/noticias-57066479. Obtenido de https://www.bbc.com/ mundo/noticias-57066479

Caícedo, M., Calvache, D., \& Jiménez, M. (2020). Vendedores informales, perspectivas de una realidad social y económica. Cali: Fundación Universitaria Católica Lumen Gentium. Obtenido de https://repository.unicatolica.edu.co/ bitstream/handle/20.500.12237/1896/ VENDEDORES_INFORMALES_ PERSPECTIVAS_REALIDAD_ SOCIAL_ECON\%C3\%93MICA. pdf?sequence=1\&isAllowed=y

Cámara de Comercio de Villavicencio. (2017). Sector comercio, análisis de los factores que afectan el desempeño economico en el centro de Villavicencio - Meta|. Villavicencio. Obtenido de http://35.224.180.91/ site/fileadmin/user_upload/2017/ Planeacion/SECTOR_COMERCIO_ VILLAVICENCIO-revMGO_2_.pdf

Cámara de Comercio de Villavicencio. (2020). Informe técnico del potencial de comerciantes vigencia 2020. Villavicencio. Obtenido de https:// s3.pagegear.co/415/76/2021/pagina_ web/informe_tecnico_potencial_ de_comerciantes_vigencia_2020_ jurisdiccion_ccv.pdf

DANE. (2019). Cuentas departamentales. Obtenido de https://www.dane.gov. co/index.php/lista-de-resultadosde-busqueda? searchword $=25 \% 20$ septiembre\%202020\&ordering=newest \&searchphrase $=$ all\&limit $=20$ 
DANE. (15 de 07 de 2021). Encuesta Mensual de Comercio (EMC). Obtenido de https://www.dane.gov.co/index.php/ estadisticas-por-tema/comercio-interno/ encuesta-mensual-de-comercio-emc

DANE. (2021). Indicador de seguimiento a la economía (ISE). Obtenido de https:// www.dane.gov.co/files/investigaciones/ boletines/pib/bol_ISE_mar2021.pdf

Departamento Nacional de Planeación. (2020). Informe municipal de seguridad $y$ convivencia ciudadana. Villavicencio. Obtenido de https://www.asocapitales. co/nueva/wp-content/uploads/2020/06/ Meta-Villavicencio.pdf

Franco, R. (11 de 09 de 2014). Advierten sobre inseguridad en el centro de Villavicencio. (E. tiempo, Entrevistador) Obtenido de https://www.eltiempo.com/archivo/ documento/CMS-14522000

Instituto Nacional de Salud. (2020). Villavicencio avanza en estudio de seroprevalencia Covid-19. Obtenido de https://www.ins.gov.co/Noticias/ Paginas/Villavicencio-avanzaen-estudio-de-seroprevalenciaCovid-19-.aspx

Orduz, T. L., \& Dimas, S. I. (2019). Factores que inciden en el empleo informal en la ciudad de Villavicencio.

Universidad Cooperativa de Colombia, Villavicencio.

Obtenido de https://repository.ucc. edu.co/bitstream/20.500.

12494/16090/10/2019

factores_inciden_empleo.pdf

Pintor, F. L., \& Guevara, V. J. (2019). Impacto socio-económicoenlacanastafamiliarde los hogares de la ciudad de Villavicencio con el incremento del IVA - Reforma
Tributaria 1819 de 2016. Universidad Cooperativa de Colombia, Villavicencio. Obtenido de https://repository.ucc.edu. co/handle/20.500.12494/12109

Rossiasco, A., \& Franco, B. (2018). Alternativas de ingresos para habitantes de calle en proceso de desarrollo personal Comunidad de vida Transitoria -Puente Aranda. Bogotá: Corporación Universitaria Uniminuto. Obtenido de https://repository.uniminuto. edu/bitstream/handle/10656/8060/ FrancoAguilarBernardo_2018. pdf?sequence $=1$ \&isAllowed $=y$

Suárez, W. (2018). No hay calle para tanto carro en Villavicencio. (E. p. Meta, Entrevistador) Villavicencio: El periódico del Meta. Obtenido de https:// periodicodelmeta.com/no-hay-callepara-tanto-carro-en-villavicencio/ 